\title{
Some Classes of Essentially Maximal Operators
}

\author{
J. TERVO
}

Suppose that $L(x, D)$ is a pseudo-differential operator with the symbol $L(x, \xi)$, that is,

$$
[L(x, D) \varphi](x)=(2 \pi)^{-n} \int_{\mathbf{R} .} L(x, \xi)(\boldsymbol{F} \varphi)(\xi) e^{i(\xi, x)} d \xi,
$$

where $\mathbf{F}$ is the Fourier transform from the Schwartz class $S$ onto $S$. The paper considers the equality of the minimal and maximal realizations of $L(x, D)$ in the $L_{2}\left(\mathbf{R}^{n}\right)$-space. Employing the symbolic calculus of Weyl sufficient criteria for the equality are proved. Also some (counter) examples for the mentioned equality are presented.

Keywords: Essential maximality, essential self-adjointness, minimal and maximal realizations AMS subject classification: 35S05, 35A35, 35A05

\section{Introduction}

Suppose that $L(x, D)$ is a pseudo-differential operator

$$
[L(x, D) \varphi](x)=(2 \pi)^{-n} \int_{\mathbf{R}^{n}} L(x, \xi)(\mathbf{F} \varphi)(\xi) e^{i(\xi, x)} d \xi .
$$

Under suitable conditions (cf. Section 2) $L(x, D)$ maps the Schwartz class $S$ into $S$ and the formal adjoint $L^{\prime}(x, D): S \mapsto S$ of $L(x, D)$ exists. When $L(x, D)$ maps $S$ into $S$ and when $L^{\prime}(x, D)$ exists we are able to define the minimal realization $\tilde{L}=L^{-*}$ and the maximal realization $L^{\prime *}$ of $L(x, D)$ in $L_{2}$. The paper seeks sufficient criteria for the equality $\dot{L}=L^{\prime *}$, that is, for the essential maximality of $L(x, D)$. In the case when $L^{\prime}=L$, the essential maximality means that $L(x, D)$ is essentially self-adjoint.

One knows several classes of operators that are essentially maximal. For partial differential operators $c f .[2-4,10,12]$. For pseudo-differential operators we refer to $[7,11,13,14]$.

Applying the Weyl calculus of pseudo-differential operators (cf. [5]), we show some additional classes of essentially maximal operators ( $\mathrm{cf}$. Theorem 3.2 and its Corollary, Theorem 3.5 and its Corollary, Theorem 3.8; cf. also Section 4). Especially, we remark that (by Corollary 3.3) the first order partial differential operators

$$
L(x, D)=\sum_{|\sigma| \leq 1} a_{\sigma}(x) D^{\sigma} \text { with }\left|D_{x}^{\alpha} a_{\sigma}(x)\right| \leq C_{o}(1+|x|)^{1-|\alpha|}
$$

are essentially maximal in $L_{2}$. One sees that the coefficients $a_{\sigma}$ may. have first degree polynomial growth in $x$.

In the last section we give various examples. The Example 4.4 shows that Corollary 3.3 is quite strict. Corollary 3.7 implies that the first order partial differential operators $L(x, D)=$

3. Tervo: University of Kuopio. Dep. Math, P. O. Box 1627, 70211 Kuopio. Finland

ISSN 0232-2064/\$2.50 (C) Heldermann Verlag Berlin 
$\sum_{|0| \leq 1} a_{\sigma}(x) D^{\sigma}$ with $C^{\infty}$-coefficients $a_{\sigma}$ obeying $\sup _{x}\left|D_{x}^{\alpha} a_{\sigma}(x)\right| \leq C_{\alpha}$ are essentially maximal. Example 4.6 shows that the corresponding result is not generally true for second order operators.

\section{Notations and preliminary notions}

2.1. Assume that $g_{(x, \xi)}: \mathbf{R}^{2 n} \mapsto \mathbf{R}$ is a positive definite quadratic form for any $(x, \xi) \in \mathbf{R}^{2 n}$. Then $g_{(x, \xi)}$ is of the form

$$
g_{(x, \xi)}(y, \eta)=\langle T(y, \eta),(y, \eta)\rangle, \quad(y, \eta) \in \mathbf{R}^{2 n},
$$

where $\langle\cdot, \cdot\rangle$ is the usual Euclidean inner product and where $T$ is a symmetric linear mapping $\mathbf{R}^{2 n} \rightarrow \mathbf{R}^{2 n}$ such that the eigenvalues of $T$ are positive. The totality $g=\left\{g_{(x, \xi)}\right\}$ of positive definite quadratic forms $g_{(x, \xi)}$ is called a Riemannian metric.

The following definitions are required (cf. [5,pp.141-179]). The Riemannian metric $g=$ $\left\{g_{(x, \xi)}\right\}$ is said to be slowly varying in $\mathbf{R}^{2 n}$ if there exist constants $\mathrm{c}, C>0$ such that $g_{(x, \xi)}(y, \eta)<$ c implies

$$
C^{-1} g_{(x, \xi)} \leq g_{(x+y, \varepsilon+\eta)} \leq C g_{(x, \xi)} .
$$

The positive weight function $m: \mathbf{R}^{2 n} \mapsto \mathbf{R}$ is $g$-continuous, if there exist constants $c, C>0$ such that $g_{(x, \xi)}(y, \eta)<\mathrm{c}$ implies

$$
C^{-1} m(x, \xi) \leq m(x+y, \xi+\eta) \leq C m(x, \xi) .
$$

Let $\sigma: \mathbf{R}^{2 n} \times \mathbf{R}^{2 n} \mapsto \mathbf{R}$ be a quadratic form (a so-called symplectic form) defined by

$$
\sigma((y, \eta),(z, \zeta))=\langle\eta, z\rangle-\langle y, \zeta\rangle
$$

For any $(x, \xi) \in \mathrm{R}^{2 n}$ we define

$$
g_{(x, \xi)}^{\sigma}(y, \eta)=\sup _{(z, \zeta) \neq 0}|\sigma((y, \eta),(z, \zeta))|^{2} / g_{(x, \xi)}(z, \zeta)
$$

The slowly: varying Riemannian metric $g$ is $\sigma$-temperate if there exist constants $C, N>0$ such that

$$
g_{(x, \xi)} \leq C g_{(y, \eta)}\left(1+g_{(x, \xi)}^{\sigma}(x-y, \xi-\eta)\right)^{N},
$$

for all $(x, \xi),(y, \eta) \in \mathbf{R}^{2 n}$. Furthermore, the $g$-continuous (weight function $m: \mathbf{R}^{2 n} \mapsto \mathbf{R}$ is $(\sigma, g)$-temperate, if there are constants $C, N>0$ such that

$$
\dot{m}(x, \xi) \leq C m(y ; \eta)\left(1+g_{(x, \xi)}^{\sigma}(x-y, \xi-\eta)\right)^{N}
$$

for all $(x, \xi),(y, \eta) \in \mathrm{R}^{2 n}$. The class $S(\dot{m}, g)$ of symbols is defined as follows: The function $L(\cdot, \cdot) \in C^{\infty}\left(\mathbf{R}^{2 n}\right)$ is in $S(m, g)$ if for any $k \in \mathrm{N}_{0}$ there exists a constant $C_{k}>0$ such that

$$
\left|L^{(k)}(x, \xi)\left(h_{1}, \ldots, h_{k}\right)\right| \leq C_{k} m(x, \xi) \prod_{j=1}^{k}\left[g_{(x, \xi)}\left(h_{j}\right)\right]^{1 / 2}
$$

for all $(x, \xi), h_{1}, \ldots, h_{k} \in \mathbf{R}^{2 n}$. Here $L^{(k)}(x, \xi)$ is the $k^{\text {th }}$ differential of $L(\cdot, \cdot)$ at $(x, \xi)$. We recall that

$$
L^{(k)}(x, \xi)\left(h_{1}, \ldots, h_{k}\right)=\sum h_{j_{1} 1} \ldots h_{j_{k} k}\left(\partial_{j_{1}} \ldots \partial_{j_{k}} L\right)(x, \xi)
$$


when $h_{j}=\left(h_{1}, \ldots, h_{2 n j}\right)$. The summation is taken over all distinct $k$-tuples $\left(j_{1}, \ldots, j_{k}\right)$ of integers between 1 and $2 n$ inclusive. $S(m, g)$ is a linear subspace of $C^{\infty}\left(\mathbf{R}^{2 n}\right)$. Furthermore, $S(m, g)$ equipped with the topology defined by the semi-norms

$$
p_{k}(L(\cdot, \cdot))=\sup _{(x, \xi) \in \mathbb{R}^{2 n}}\left[\sup _{h, \in \mathbb{R}^{2 n}-\{0\}}\left|L^{(k)}(x, \xi)\left(h_{1}, \ldots, h_{k}\right)\right| / \prod_{j=1}^{k}\left[g_{(x, \xi)}(h,)\right]^{1 / 2}\right] / m(x, \xi)
$$

is a Fréchet space.

Remark 2.1. (A) One has the topological inclusions $C_{0}^{\infty}\left(\mathbf{R}^{2 n}\right) \subset S(m, g) \subset C^{\infty}\left(\mathbf{R}^{2 n}\right)$, when $C_{0}^{\infty}\left(\mathbf{R}^{2 n}\right)$ and $C^{\infty}\left(\mathbf{R}^{2 n}\right)$ are equipped with the standard locally convex topologies. (B) Assume that $L_{1}(\cdot, \cdot) \in S\left(m_{1}, g\right)$ and $L_{2}(\cdot, \cdot) \in S\left(m_{2}, g\right)$. Then $\left(L_{1} L_{2}\right)(\cdot, \cdot)=L_{1}(\cdot, \cdot) L_{2}(\cdot, \cdot) \in S\left(m_{1} m_{2}, g\right)$. (C) $S\left(m_{1}, g\right) \subset S\left(m_{2}, g\right)$ if and only if $m_{1} \leq C m_{2}$.

2.2 In this subsection we deal with a Riemannian metric of the special separated form

$$
g_{(x . \xi)}(y, \eta)=\frac{|y|^{2}}{g_{1}^{2}(x, \xi)}+\frac{|\eta|^{2}}{g_{2}^{2}(x, \xi)},
$$

where $g_{j}: \mathbf{R}^{2 n} \mapsto \mathbf{R}$ are positive functions. This kind of metric occurs often in applications. One has the following theorem in which

$$
U_{(x, \xi), c}=\left\{(y, \eta) \in \mathbf{R}^{2 n}|| x-y \mid<c g_{1}(x, \xi) \text { and }|\xi-\eta|<c g_{2}(x, \xi)\right\} .
$$

Theorem 2.2. Let $g=\left\{g_{(x, \varepsilon)}\right\}$ be a Riemannian metric such that $g_{(x, \varepsilon)}$ has the form $(2.6)$. Then:

(i) The metric $g$ is slowly varying if and only if there exist constants c, $C>0$ such that

$$
C^{-1} g_{j}(x, \xi) \leq g_{j}(y, \eta) \leq C g_{j}(x, \xi) \quad(j=1,2)
$$

for all $(x, \xi) \in \mathbf{R}^{2 n}$ and $(y, \eta) \in U_{(x, \xi) . c}$.

(ii) The weight function $m: \mathbf{R}^{2 n} \mapsto \mathbf{R}$ is $g$-continuous if and only if there exist constants c, $C>0$ such that

$$
C^{-1} m(x, \xi) \leq m(y, \eta) \leq C m(x, \xi)
$$

for all $(x, \xi) \in \mathbf{R}^{2 n}$ and $(y, \eta) \in U_{(x, \xi), c}$.

(iii) The metric $g$ is $\sigma$-temperate if and only if there exist constants $C, N>0$ such that

$$
g_{j}(y, \eta) / g_{j}(x, \xi) \leq C\left(1+g_{2}^{2}(x, \xi)|x-y|^{2}+g_{1}^{2}(x, \xi)|\xi-\eta|^{2}\right)^{N / 2} \quad(j=1,2)
$$

for all $(x, \xi),(y, \eta) \in \mathbf{R}^{2 n}$.

(iv) The weight function $m: \mathbf{R}^{2 n} \mapsto \mathbf{R}$ is $(\sigma, g)$-temperate if and only if there exist constants $C, N>0$ such that

$$
m(x, \xi) \leq C m(y, \eta)\left(1+g_{2}^{2}(x, \xi)|x-y|^{2}+g_{1}^{2}(x, \xi)|\xi-\eta|^{2}\right)^{N / 2}
$$

for all $(x, \xi),(y, \eta) \in \mathbf{R}^{2 n}$.

(v) The symbol $L(\cdot, \cdot) \in C^{\infty}\left(\mathbf{R}^{2 n}\right)$ lies in the symbol class $S(m, g)$ if and only if for any $\alpha, \beta \in \mathrm{N}_{0}^{n}$ there exists a constant $C_{\alpha, \beta}$ such that

$$
\left|D_{x}^{o} D_{\xi}^{\beta} L(x, \xi)\right| \leq C_{\alpha \beta} m(x, \xi) g_{1}^{-|\alpha|}(x, \xi) g_{2}^{-|\rho|}(x, \xi)
$$


for all $(x, \xi) \in \mathbf{R}^{2 n}$.

Proof. The validity of the claims (i), (ii) and (v) can be obtained by simple conclusions (which are omitted). The claims (iii) and (iv) follow easily, if one verifies that

$$
g_{(x, \xi)}^{\sigma}(y, \eta)=g_{2}^{2}(x, \xi)|y|^{2}+g_{1}^{2}(x, \xi)|\eta|^{2} .
$$

We consider the relation (2.7). One sees that, with $\zeta=-y g_{2}^{2}(x, \xi)$ and $z=\eta g_{1}^{2}(x, \xi)$,

$$
|\sigma((y, \eta),(z, \zeta))|^{2}=|\eta|^{2} g_{1}^{2}(x, \xi)+|y|^{2} g_{2}^{2}(x, \xi)
$$

and then

$$
\begin{aligned}
g_{(x, \xi)}^{\sigma}(y, \eta) & \geq|\sigma((y, \eta),(z, \zeta))|^{2} /\left(\frac{|z|^{2}}{g_{1}^{2}(x, \xi)}+\frac{|\zeta|^{2}}{g_{2}^{2}(x, \xi)}\right) \\
& =\frac{\left(|\eta|^{2} g_{1}^{2}(x, \xi)+|y|^{2} g_{2}^{2}(x, \xi)\right)^{2}}{|\eta|^{2} g_{1}^{2}(x, \xi)+|y|^{2} g_{2}^{2}(x, \xi)}=g_{2}^{2}(x, \xi)|y|^{2}+g_{1}^{2}(x, \xi)|\eta|^{2} .
\end{aligned}
$$

The converse inequality ${ }^{n} \leq^{n}$ of (2.7) is easily seen

Remark 2.3. Suppose that the Riemannian metric (2.6) is slowly varying. Then the weight function $m=g_{1}^{R} g_{2}^{r}$ is $g$-continuous, for any $R, r \in \mathrm{R}$. The proof follows easily from Theorem 2.2. Similary, one sees that when $g$ is $\sigma$-temperate, then $m=g_{1}^{R} g_{2}^{r}$ is $(\sigma, g)$-temperate.

Example 2.4. (A) Consider the case where

$$
g_{1}^{2}(x, \xi)=\left(1+|\xi|^{2}\right)^{\delta}\left(1+|x|^{2}\right)^{\delta^{\prime}} \quad \text { and } \quad g_{2}^{2}(x, \xi)=\left(1+|\xi|^{2}\right)^{\rho}\left(1+|x|^{2}\right)^{\rho^{\prime}}
$$

Suppose that $\delta \leq 0, \delta^{\prime} \leq 1$ and $\rho \leq 1, \rho^{\prime} \leq 0$. Then the metric

$$
g_{(x, \xi)}(y, \eta)=\frac{|y|^{2}}{\left(1+|\xi|^{2}\right)^{\delta}\left(1+|x|^{2}\right)^{\delta^{\prime}}}+\frac{|\eta|^{2}}{\left(1+|\xi|^{2}\right)^{\rho}\left(1+|x|^{2}\right)^{\rho^{\prime}}}
$$
is slowly varying: Choose in Theorem $2.2 / \mathrm{Part}(\mathrm{i}) \mathrm{c}=\frac{1}{2}$ and assume that $(y, \dot{\eta}) \in U_{(x, \xi), \frac{1}{2}}$. Then
we obtain

$$
4|x-y|^{2}<g_{1}^{2}(x, \xi)=\left(1+|\xi|^{2}\right)^{\delta}\left(1+|x|^{2}\right)^{\delta^{\prime}} \leq\left(1+|x|^{2}\right)
$$

and so $\frac{1}{4}\left(1+|x|^{2}\right) \leq 1+|y|^{2} \leq 4\left(1+|x|^{2}\right)$. Similary, one finds that $1\left(1+|\xi|^{2}\right) \leq 1+|\eta|^{2} \leq 4\left(1+|\xi|^{2}\right)$ for any $(y, \eta) \in U_{(x, \xi), \frac{1}{2}}$. Hence it is easy to see that $C^{-1} g_{j}(x, \xi) \leq g_{j}(y, \eta) \leq C g_{j}(x, \xi)$ for all $(y, \eta) \in U_{(x, \xi), \frac{1}{2}}$ with a suitable constant $C>0$. (B) Due to Remark 2.3 the weight function $m(x, \xi)=\left(1+|\xi|^{2}\right)^{\delta R+o r}\left(1+|x|^{2}\right)^{\delta^{\prime} R+\rho^{\prime} r}$ is $g$-continuous for any $R, r \in \mathbf{R}$ and $\delta \leq 0, \delta^{\prime} \leq 1, \rho \leq$ $1, \rho^{\prime} \leq 0$. Especially, one can choose $\delta=\rho^{\prime}=0$ and $\rho=\delta^{\prime}=1$. In this case, for example, $L(\cdot, \cdot) \in C^{\infty}\left(\mathbf{R}^{2 n}\right)$ belongs to $S(m, g)$ if and only if

$$
\left|\left(D_{x}^{\alpha} D_{\xi}^{\mathcal{\beta}} L\right)(x, \xi)\right| \leq C_{\alpha, \beta}\left(1+|x|^{2}\right)^{R}\left(1+|\xi|^{2}\right)^{r}\left(1+|x|^{2}\right)^{-|\alpha|}\left(1+|\xi|^{2}\right)^{-|\mathcal{|}|} .
$$

It is remarkable to note that the right-hand side may increase polynomially in $x$. (C) In the case when $\delta^{\prime}=\rho^{\prime}=0, \delta \leq 0, \rho \leq 1$ and $m(x, \xi)=(1+|\xi|)^{r / 2}$, one sees that $S(m, g)$ is essentially the Hörmander class $S_{-\delta, \rho}^{r}$ of symbols.

Later we shall consider also the $\sigma$-temperate criterion for the metric (2.8). 
2.3 Let $g$ and $m$ be as in the Subsection 2.1. Choose $L(\cdot, \cdot)$ from $S(m, g)$. Define a pseudodifferential operator $L(x, D)$ by the formula

$$
[L(x, D) \varphi](x)=(2 \pi)^{-n} \int_{\mathbf{R}^{n}} L(x, \xi)(\mathbf{F} \varphi)(\xi) e^{i(\xi, x)} d \xi,
$$

where $\mathbf{F}$ is the Fourier transform from the Schwartz class $S$ into $S$ and where $\varphi$ belongs to $S$. Denote by $h: \mathbf{R}^{2 n} \rightarrow \mathbf{R}$ the positive function defined by

$$
h^{2}(x, \xi)=\sup _{(y, \eta) \neq 0}\left[g_{(x, \xi)}(y, \eta) / g_{(x, \xi)}^{\sigma}(y, \eta)\right] .
$$

In the sequel we shall assume that $h \leq 1$. In the case when $g$ is the separated form (2.6), the function $h$ is $1 / g_{1} g_{2}$ (cf. (2.7)) and then the condition

$$
g_{1} g_{2} \geq 1
$$

implies that $h \leq 1$. We need the following results of [5], which contain some essential tools concerning the operators (2.9) (for some special case $\mathrm{cf}$. also [1]).

Theorem 2.5. Suppose that Riemannian metric $g$ is $\sigma$-temperate,

$$
g_{(x, \xi)}(y, \eta)=g_{(x, \xi)}(y,-\eta) \text { for all }(x, \xi),(y, \eta) \in \mathbf{R}^{2 n},
$$

the weight function $m$ is $(\sigma, g)$-temperate and the symbol $L(\cdot, \cdot) \in S(m, g)$. Then the operator $L(x, D)$ defined by (2.9) maps $S$ continuously into $S$ (in $S$ we use the standand Fréchet space topology).

We say that a linear operator $L^{\prime}(x, D): S \mapsto S$ is the formal adjoint of $L(x, D)$ if one has

$$
\langle\varphi, L(x, D) \psi\rangle_{0}=\left\langle L^{\prime}(x, D) \varphi, \psi\right\rangle_{0} \text { for all } \varphi, \psi \in S \text {. }
$$

Here $\langle\cdot, \cdot\rangle_{0}$ denotes the $L_{2}$ inner product, that is, $\langle u, v\rangle_{0}=\int_{\mathbb{R}^{n}} u(x) \overline{v(x)} d x$. One has

Theorem 2.6. Suppose that the Riemannian metric $g$ is $\sigma$-temperate, (2.11) is valid, the weight function $m$ is $(\sigma, g)$-temperate and that the symbol $L(\cdot, \cdot) \in S(m, g)$. Furthermore, assume that

$$
h \leq 1 \text {. }
$$

Then the formal adjoint $L^{\prime}(x, D): S \mapsto S$ of $L(x, D)$ exists. In addition,

$$
\left[L^{\prime}(x, D) \varphi\right](x)=(2 \pi)^{-n} \int_{\mathbf{R}^{n}} L^{\prime}(x, \xi)(\mathbf{F} \varphi)(\xi) e^{i(\xi, x)} d \xi \quad \text { for all } \varphi \in S,
$$

where, for any $N \in \mathrm{N}_{0}, L^{\prime}(x, \xi)$ has the decomposition

$$
L^{\prime}(x, \xi)=\overline{L(x, \xi)}+\sum_{0<|\alpha|<N} \frac{(-i)^{|\alpha|}}{\alpha !} \overline{\left(\partial_{\xi}^{\alpha} \partial_{x}^{\alpha} L\right)(x, \xi)}+R_{N}(x, \xi)
$$

with $R_{N}(\cdot, \cdot) \in S\left(h^{N} m, g\right)$.

Theorem 2.7. Suppose that the Riemannian metric $g$ is $\sigma$-temperate, (2.11) is valid, the weight function $m$ is $(\sigma, g)$-temperate and that $h \leq 1$. Furthermone, assume that $m \leq C$ and that 
$\left\{L_{j}(\cdot, \cdot)\right\} \subset S(m, g)$ is a bounded sequence of symbols (that is, $\left\{p_{k}\left(L_{j}(\cdot, \cdot)\right) \mid j \in \mathrm{N}_{0}\right\}$ is bounded for any $k \in \mathrm{N}_{0}$ ). Then there exists a constant $C>0$ such that

$$
\left\|L_{j}(x, D) \varphi\right\|_{0} \leq C\|\varphi\|_{0} \quad \text { for all } \varphi \in S, j \in \mathrm{N}_{0} .
$$

Theorem 2.8. Suppose that the Riemannian metric $g$ is $\sigma$-temperate, (2.11) is valid, the weight functions $m_{1}$ and $m_{2}$ are $(\sigma, g)$-temperate and that $h \leq 1$. Futhermore, assume that the symbol $L(\cdot, \cdot) \in S\left(m_{1}, g\right)$ and that $\left\{L_{j}(\cdot, \cdot)\right\} \subset S\left(m_{2}, g\right)$ is a bounded sequence of symbols. Then the composition $L(x, D) \circ L_{j}(x, D)$ has the form

$$
\left[\left(L(x, D) \circ L_{j}(x, D)\right) \varphi\right](x)=(2 \pi)^{-n} \int_{\mathbf{R}^{n}}\left(L \circ L_{j}\right)(x, \xi)(\mathbf{F} \varphi)(\xi) e^{i(\xi, x)} d \xi \text { for all } \varphi \in S,
$$

where the symbol $\left(L \circ L_{j}\right)(\cdot, \cdot) \in S\left(m_{1} m_{2}, g\right)$. In addition, one has, for any $N \in \mathbf{N}_{0}$,

$$
\begin{aligned}
\left(L \circ L_{j}\right)(x, \xi)=L(x, \xi) L_{j}(x, \xi) & +\sum_{0<|\alpha|<N} \frac{(-i)^{|\alpha|}}{\alpha !} \partial_{\xi}^{\alpha} L(x, \xi) \partial_{x}^{\alpha} L_{j}(x, \xi) \\
& +R_{j, N}(x, \xi)
\end{aligned}
$$

where $\left\{R_{j, N}(\cdot, \cdot)\right\}$ is a bounded set of symbols in $S\left(m_{1} m_{2} h^{N}, g\right)$.

2.4 Suppose that $L: S \mapsto S$ is a (continuous) linear operator such that the formal adjoint $L^{\prime}: S \rightarrow S$ of $L$ exists, that is, $\langle\varphi, L \psi\rangle_{0}=\left\langle L^{\prime} \varphi, \psi\right\rangle_{0}$ for all $\varphi, \psi \in S$. We shall define two extensions of $L$ in the $L_{2}$-space. Define a (dense) linear operator

$$
L_{0}: L_{2} \rightarrow L_{2}, \quad L_{0 \varphi}=L_{\varphi} \text { for } \varphi \in D\left(L_{0}\right):=S .
$$

One sees that

$$
\left\langle\varphi, L_{0} \psi\right\rangle_{0}=\langle\varphi, L \psi\rangle_{0}=\left\langle L^{\prime} \varphi, \psi\right\rangle_{0}=\left\langle L_{0}^{\prime} \varphi, \psi\right\rangle_{0},
$$

and so $S \subset D\left(L_{0}^{*}\right)$ and $L_{0}^{*} \varphi=L^{\prime} \varphi$ for $\varphi \in S$. Here $L_{0}^{*}$ is the $L_{2}$-adjoint of $L_{0}$. Since $D\left(L_{0}^{*}\right)$ is dense in $L_{2}$, one gets that $L_{0}$ is a closable operator and so the smallest closed extension $\tilde{L}: L_{2} \rightarrow L_{2}$ of $L_{0}$ exists. We recall that $D(\tilde{L})=\left\{u \in L_{2} \mid \varphi_{n} \rightarrow u, L_{0} \varphi_{n} \rightarrow f\right.$ for some $\left.f \in L_{2},\left\{\varphi_{n}\right\} \subset S\right\}$ and $\dot{L} u=f$. One knows also that $\dot{L}=\dot{L}_{0}^{*-}$, where $L_{0}^{-*}$ is the $L_{2}$ adjoint of $L_{0}^{*}$ (cf. $[6$, p. 168]).

Since $\left(L^{\prime}\right)^{\prime}=L$, one can similarly define $L_{0}^{\prime-}$ and $L^{\prime}$. In the sequel we denote $L^{\prime *}=L_{0}^{\prime *}$ and $L^{*}=L_{0}^{*}$. The above conclusions show that $L \subset \bar{L}$ and $\bar{L} \subset L^{\prime *}$. Hence $\bar{L}$ and $L^{\prime *}$ are (closed) extensions of $L$. The operators $\bar{L}$ and $L^{\prime \prime}$ are called a minimal and maximal $L_{2}$-realization of $L$, respectively. In the case when $\tilde{L}=L^{\prime *}$ we say that $L$ is essentially maximal in $L_{2}$. When $L=L^{\prime}$ the essential maximality means that $L$ is essentially self-adjoint.

\section{On essential maximality of operators whose symbol lie in $S(m, g)$}

3.1 Let $\theta$ be in $C_{0}^{\infty}$ such that $\theta \geq 1$ and $\theta(\xi)=1$ for all $|\xi| \leq 1$. Define $\theta_{j} \in C_{0}^{\infty}\left(\mathbf{R}^{n}\right)$ and $\psi_{j} \in C_{0}^{\infty}\left(\mathbf{R}^{2 n}\right)$ by the relations $\theta_{j}(\xi)=\theta(\xi / j)$ and $\psi_{j}(x, \xi)=\theta_{j}(\xi) \theta_{j}(x)$, respectively. Let $\theta_{j}(D)$ and $\psi_{j}(x, D)$ be the corresponding pseudo-differential operators with symbols $\theta_{j}(x, \xi) \equiv$ $\theta_{j}(\xi)$ and $\psi_{j}(x, \xi)$. Denote by $\theta_{j}$ and $\Psi_{j}$ the operators $\theta_{j}^{\prime *}$ and $\psi_{j}^{\prime *}$, respectively. For any $u \in L_{2}$ one observes that $F\left(\theta_{j} u\right)(\xi)=\theta_{j}(\xi)(F u)(\xi)$ and so $\Theta_{j} u \in \bigcap_{0 \geq 0} H_{k} \subset C^{\infty}$ where 
$H_{k_{j}}=\left\{u \in L_{2} \mid\left(1+|\xi|^{2}\right)^{\bullet / 2}(F u)(\xi) \in L_{2}\right\}$. Furthermore, one sees that $\Psi_{j} u=\theta_{j} \Theta_{j} u$ and so $\Psi_{j} u \in C_{0}^{\infty}$, for any $u \in L_{2}$ (in fact it is easy to see that $\Psi_{j} u=\theta_{j}\left(u * \theta_{j}\right)$ ). Since $\theta_{j}(\xi) \rightarrow 1$ for any $\xi \in \mathbf{R}^{n}$ and since $\left|\theta_{j}(\xi)\right| \leq \sup _{\xi}|\theta(\xi)|$ one gets (due to the Dominated Convergence Theorem)

$$
\begin{aligned}
\left\|\theta_{j} u-u\right\|_{0} & =\left((2 \pi)^{-n} \int_{\mathbb{R}^{n}}|F(\theta ; u)(\xi)-(F u)(\xi)|^{2} d \xi\right)^{1 / 2} \\
& =\left((2 \pi)^{-n} \int_{R^{n}}|(\theta ;(\xi)-1)(F u)(\xi)|^{2} d \xi\right)^{1 / 2} \rightarrow 0
\end{aligned}
$$

with $j \rightarrow \infty$. Then we also get

$$
\|\Psi ; u-u\|_{0} \leq\left\|\theta_{j} \theta_{j} u-\theta_{j} u\right\|_{0}+\left\|\theta_{j} u-u\right\|_{0} \rightarrow 0
$$

with $j \rightarrow \infty$.

Lemma 3.1. Suppose that $g$ is a Riemannian metric such that, with $\mathrm{c}>0$,

$$
g_{(x, \xi)}(y, \eta) \geq c|(y, \eta)|^{2} /(1+|x|+|\xi|)^{2} \quad \text { for all }(x, \xi),(y, \eta) \in \mathbf{R}^{2 n} .
$$

Futhermore assume that $m: \mathbf{R}^{2 n} \rightarrow \mathbf{R}$ is a weight function obeying, with $c>0$,

$$
m(x, \xi) \geq c \quad, \text { for all }(x, \xi) \in R^{2 n} .
$$

Then the sequence $\left\{\psi_{j}(\cdot, \cdot)\right\}$ of symbols is bounded in $S(m, g)$.

Proof. Let $\tilde{\theta} \in C_{0}^{\infty}\left(\mathbf{R}^{2 n}\right)$ such that $\bar{\theta}(x, \xi)=\theta(x) \theta(\xi)$. Then $\psi_{j}(x, \xi)=\dot{\theta}((x, \xi) / j)$. For any $(\alpha, \beta) \in \mathbf{N}_{0}^{2 n}$ there exists a constant $C_{\alpha, \beta}>0$ such that

$$
(1+|x|+|\xi|)^{|\alpha|+|\beta|}\left|\left(D_{\xi}^{\alpha} D_{\xi}^{\beta} \tilde{\theta}\right)(x, \xi)\right| \leq C_{\alpha \beta \beta}
$$

and so

$$
\begin{aligned}
\left|\left(D_{x}^{\alpha} D_{\xi}^{\beta} \psi_{j}\right)(x, \xi)\right| & =\frac{1}{j^{|\alpha| j^{|\beta|} \mid}\left|\left(D_{x}^{\alpha} D_{\xi}^{\beta} \bar{\theta}\right)((x, \xi) / j)\right|} \\
& \leq C_{\alpha, \beta} \frac{1}{j^{|\alpha|+|\beta|}}\left(1+\left|\frac{x}{j}\right|+\left|\frac{\xi}{j}\right|\right)^{-(|\alpha|+|\beta|)} \\
& \leq C_{\alpha, \beta}(1+|x|+|\xi|)^{-(|\alpha|+|\rho|)}
\end{aligned}
$$

Thus we obtain, for any $k \in \dot{N}$,

$$
\begin{aligned}
\left|\psi_{j}^{(k)}(x, \xi)\left(h_{1}, \ldots, h_{k}\right)\right| & \leq \sum\left|h_{j_{1} 1}\right| \ldots\left|h_{j_{k} k}\right|\left|\left(\partial_{j_{1}} \ldots \partial_{j_{k}} \psi_{j}\right)(x, \xi)\right| \\
& \leq C_{k}\left|h_{j_{1} 1}\right| \ldots\left|h_{j_{k} k}\right|(1+|x| \dot{+}+\xi \mid)^{-k} \\
& \leq C_{k}\left|h_{1}\right| \ldots\left|h_{k}\right|(1+|x|+|\xi|)^{-k} \\
& \leq C_{k} \mathrm{c}^{-k / 2} g_{(x, \xi)}^{1 / 2}\left(h_{1}\right) \ldots g_{(x, \xi)}^{1 / 2}\left(h_{k}\right) \\
& \leq C_{k} \mathrm{c}^{-(k / 2)-1} m(x, \xi) \prod_{j=1}^{k} g_{(x, \xi)}^{1 / 2}\left(h_{j}\right)
\end{aligned}
$$

which implies the assertion 
We shall now state

Theorem 3.2. Suppose that the Riemannian metric ?

$$
g_{(x, \xi)}(y, \eta)=\frac{|y|^{2}}{g_{1}^{2}(x, \xi)}+\frac{|\eta|^{2}}{g_{2}^{2}(x, \xi)}
$$

is o-temperate and that there exist constants $\mathrm{c}, C>0$ such that

$$
g_{1} g_{2} \geq \mathrm{c}
$$

and

$$
g_{j}(x, \xi) \leq C(1+|x|+|\dot{\xi}|) \quad(j=1,2) .
$$

Furthermore, suppose that $m$ is $a(\sigma, g)$-temperate weight function such that

$$
h m \leq C \text {. }
$$

Let $L(\cdot, \cdot)$ be a symbol of the class $S(m, g)$. Then the operator $L(x, D)$ is essentially maximal.

Proof. Due to Theorem 2.8 (note that $(2.11)$ is valid and that $h \leq \frac{1}{g_{192}} \leq \mathrm{c}^{-1}$ ) and to Lemma 3.1 (in which we choose $m(x, \xi) \equiv 1$ ) we find that

$$
\left(\psi_{j} \circ L\right)(x, \xi)=\psi_{j}(x, \xi) L(x, \xi)+R_{j}^{\prime}(x, \xi)
$$

and

$$
\left(L \circ \psi_{j}\right)(x, \xi)=L(x, \xi) \psi_{j}(x, \xi)+R_{j .}^{\prime \prime}(x, \xi)
$$

where $\left\{R_{j}^{\prime}(\cdot, \cdot)\right\}$ and $\left\{R_{j}^{\prime \prime}(\cdot, \cdot)\right\}$ are bounded in $S(m h, g)$. Hence by Theorem 2.7 and because $h m \leq C$,

$$
\left(\psi_{j} \circ L\right)(x, D)=\left(L \circ \psi_{j}\right)(x, D)+R_{j}(x, D),
$$

where

$$
\left\|R_{j}(x, D) \varphi\right\|_{0} \leq C\left\|_{\varphi}\right\|_{0} \text { for all } j \in \mathrm{N}, \varphi \in S .
$$

Let $u \in D\left(L^{\prime *}\right)$. Then we find that

$$
\begin{aligned}
\left\langle\Psi_{j}\left(L^{\prime *} u\right), \varphi\right\rangle_{0} & =\left\langle L^{\prime *} u, \psi_{j}^{\prime}(x, D) \varphi\right\rangle_{0} \\
& =\left\langle u,\left(L^{\prime} \circ \psi_{j}^{\prime}\right)(x ; D) \varphi\right\rangle_{0} \\
& =\left\langle u,\left(\psi_{j} \circ L\right)^{\prime}(x, D) \varphi\right\rangle_{0} \\
& =\left\langle u,\left(L \circ \psi_{j}\right)^{\prime}(x, D) \varphi\right\rangle_{0}+\left\langle u, R_{j}^{\prime}(x, D) \varphi\right\rangle_{0} \\
& =\left\langle L^{\prime \bullet}\left(\Psi_{j} u\right), \varphi\right\rangle_{0}+(R ; u, \varphi\rangle_{0}
\end{aligned}
$$

for all $\varphi \in S$ where $R_{j}$ is the continuous extension of $R_{j}(x, D)$ (cf.( 3.7$)$ ). Thus we get

$$
L(x, D)(\Psi, u)=\Psi_{j}\left(L^{\prime \bullet} u\right)-R ; u .
$$

In virtue of (3.7) we find that (cf. also (3.1))

$$
\left\|L(x, D)\left(\Psi_{j} \underline{u}\right)\right\|_{0} \leq\left\|\Psi_{j}\left(L^{\prime \bullet} u\right)\right\|_{0}+\left\|R_{j} u\right\|_{0} \leq C^{\prime}\left(\left\|L^{\prime \bullet} u\right\|_{0}+\|u\|_{0}\right)
$$


for any $u \in D\left(L^{\prime-}\right), j \in \mathrm{N}_{0}$. The Banach-Saks Theorem implies that there exists a subsequence $\left\{\Psi_{j k} u\right\}$ of $\left\{\Psi_{j} u\right\}$ such that

$$
\left\|l^{-1} \sum_{k=1}^{l} L(x, D)\left(\Psi_{j_{k}} u\right)-f\right\|_{0}=\left\|L(x, D)\left(1^{-1} \sum_{k=1}^{1} \Psi_{j_{k}} u\right)-f\right\|_{0} \rightarrow 0 \quad(l \rightarrow \infty)
$$

with some $f \in L_{2}$. In addition by (3.1) we see that $\left\|l^{-1} \sum_{k=1}^{l} \Psi_{j_{k}} u-u\right\|_{0} \rightarrow 0$ with $l \rightarrow \infty$. Hence $u \in D(\bar{L})$ and $\bar{L} u=f \quad\left(=L^{\prime-} u\right)$, which completes the proof

For the first order partial differential operators we obtain the next corollary.

Corollary 3.3. Let $L(x, D)=\sum_{|\sigma| \leq 1} a_{\sigma}(x) D^{\sigma}$ be a first onder partial differential operator such that $a_{\sigma} \in C^{\infty}$ and that

$$
\left|D_{x}^{a} a_{\sigma}(x)\right| \leq C_{\alpha}(1+|x|)^{1-|a|} \text { for }|\sigma| \leq 1, a \in \mathbf{N}_{0}^{n} x \in \mathbf{R}^{n} .
$$

Then $L(x, D)$ is essentially maximal.

Proof. We choose the Riemannian metric

$$
g_{(x, \xi)}(y, \eta)=\frac{|y|^{2}}{(1+|x|)^{2}}+\frac{|\eta|^{2}}{(1+|\xi|)^{2}}
$$

Due to Example 2.4, $g$ is slowly varying. Remark 2.3 implies that the weight function $m(x, \xi)=$ $(1+|x|)(1+|\xi|)$ is $g$-continuous.

We verify that $g$ is $\sigma$-temperate. Due to Theorem 2.2 one must verify that

$$
\frac{1+|y|}{1+|x|} \leq C(1+(1+|\xi|)|x-y|+(1+|x|)|\xi-\eta|)^{N}
$$

and

$$
\frac{1+|\eta|}{1+|\xi|} \leq C(1+(1+|\xi|)|x-y|+(1+|x|)|\xi-\eta|)^{N}
$$

Since

$$
1+|y| \leq 1+|x|+|y-x| \leq(1+|x|)(1+|x-y|), \quad 1+|\eta| \leq(1+|\xi|)(1+|\xi-\eta|)
$$

the conditions (3.9) - (3.10) hold with $N=1$. Thus $g$ is $\sigma$-temperate.

Theorem 2.2 implies that $m$ is $(\sigma, g)$-temperate, if

$$
\frac{(1+|x|)}{1+|y|} \frac{(1+|\xi|)}{1+|\eta|} \leq C(1+(1+|\xi|)|x-y|+(1+|x|)|\xi-\eta|)^{N} .
$$

One sees that

$$
\frac{1+|x|}{1+|y|} \frac{1+|\xi|}{1+|\eta|} \leq(1+|x-y|)(1+|\xi-\eta|)
$$

and so (3.11) is valid with $N=2$. Thus $m$ is $(\sigma, g)$-temperate.

The assumptions (3.2) - (3.3) of Theorem 3.2 are trivial and by. (3.8) one sees that $L(\cdot, \cdot) \in$ $S(m, g)$. Noting that $h m \leq \frac{1}{g_{193}} m=1$ one may conclude the assertion from Theorem 3.2

Remark 3.4. From Theorem 3.2 one can conclude more general results like those given in Corollary 3.3; for example one may consider pseudo-differential operators with symbol $L(x, \xi)$ obeying

$$
\left|\left(D_{x}^{a} D_{\xi}^{\rho} L\right)(x, \xi)\right| \leq C_{\alpha, \beta}(1+|x|)^{1-|\alpha|}(1+|\xi|)^{1-|\beta|} .
$$


3.2 In this subsection we consider the case $L(x, \xi)=L_{0}(\xi)+P(x, \xi)$ where $L_{0}(\xi) \equiv L_{0}(x, \xi) \epsilon$ $S(\tilde{m}, g)$ and where $P(x, \xi)$ is related to the first order operators.

Theorem 3.5 Suppose that the Riemannian metric

$$
\dot{g}_{(x, \xi)}(y, \eta)=\frac{|y|^{2}}{g_{1}^{2}(x, \xi)}+\frac{|\eta|^{2}}{g_{2}^{2}(x, \xi)}
$$

is $\sigma$-temperate, where $g_{1}$ and $g_{2}$ satisfy (3.2) and

$$
g_{j}(x, \xi) \leq C(1+|\xi|) \quad(j=1,2) .
$$

Furthermore, let $m$ and $\bar{m}$ be $(\sigma, g)$-temperate weight functions such that (for $m)(3.4)$ is valid and that the symbols $L_{0}(\cdot) \in S(\tilde{m}, g), P(\cdot, \cdot) \in S(m, g)$. Then the operator $L(x, D)=L_{0}(D)+P(x, D)$ is essentially maximal.

Proof. One sees that

$$
\theta_{j}(D)(L(x, D) \varphi)=\theta_{j}(D)\left(L_{0}(D) \varphi\right)+\theta_{j}(D)(P(x, D) \varphi) .
$$

Similarly as in Lemma 3.1 we get from (3.12) and from (3.4) that $\left\{\theta_{j}(\cdot)\right\}$ is bounded in $S(1, g)$. Futhermore, by Theorem 2.8

$$
\left(\theta_{j} \circ P\right)(x, \xi)=\theta_{j}(\xi) P(x, \xi)+R_{j}(x, \xi),
$$

where $\left\{R_{j}(\cdot, \cdot)\right\}$ is bounded in $S(m h, g) \subset S(1, g)$. Hence

$$
\theta_{j}(D)(L(x, D) \varphi)=L(x, D)\left(\theta_{j}(D) \varphi\right)+R_{j}(x, D) \varphi,
$$

which implies

$$
\theta_{j}\left(L^{\prime *} u\right)=L^{\prime *}\left(\Theta_{j} u\right)+R_{j} u \quad \text { for } u \in D\left(L^{\prime *}\right) .
$$

From (2.4), (2.7) and (3.12) we obtain

$$
\begin{aligned}
\left|L_{0}(\xi)\right| & \leq C \bar{m}(0, \xi) \leq C^{\prime} \bar{m}(0,0)\left(1+g_{(0, \xi)}^{\sigma}(0, \xi)\right)^{N} \\
& \leq C^{\prime \prime}\left(1+g_{1}^{2}(0, \xi)|\xi|^{2}\right)^{N} \leq C^{\prime \prime \prime}\left(1+|\xi|^{2}\right)^{2 N}
\end{aligned}
$$

and so $\left\|L_{0}(D) \varphi\right\|_{0} \leq C\|\varphi\|_{k_{4} N}$ (recall that $k_{3}(\xi)=\left(1+|\xi|^{2}\right)^{\circ / 2}$ ). Due to (3.4) we see that $h \leq$ $C / m$ and, by $(3.12),(1+|\xi|)^{-|\beta|} \leq C^{|\beta|} g_{2}^{-|\beta|}(x, \xi)$. Thus one easily gets (by (3.12)) that $k_{-2}(\cdot) \epsilon$ $S\left(\frac{1}{m}, g\right)$. Hence $\left(P \circ k_{-2}\right)(\cdot, \cdot) \in S(1, g)$ and then, by Theorem $2.7,\left\|P(x, D)\left(k_{-2}(D) \varphi\right)\right\|_{0} \leq$ $C\|\varphi\|_{0}$ which implies $\|P(x, D) \varphi\|_{0} \leq C\|\varphi\|_{k_{2}}$. The above inequalities imply that (with $s:=$ $4 N+2)$

$$
\|L(x, D) \varphi\|_{0} \leq C\|\varphi\|_{k_{s}} \text {, for all } \varphi \in S
$$

and then $H_{k,} \subset D(\bar{L})$. Since $\Theta_{j} u \in H_{k,}$ one gets from (3.13)

$$
\left\|\bar{L}\left(\theta_{j} u\right)\right\|_{0} \leq C\left(\left\|L^{\prime *} u\right\|_{0}+\|u\|_{0}\right) \text { for } u \in D\left(L^{\prime *}\right)
$$

from which it follows (as above) that $u \in D(\bar{L})$ and that $\bar{L}_{u}=L^{\prime \prime} u$, as desired $I$

Remark 3.6. The content of Theorem 3.5 can be formulated also for more general metrics. We omit this generalization. 
Corollary 3.7. Let $L(x, D)=L_{0}(D)+P(x, D)$ be a linear partial differential operator, where $L_{0}(D)=\sum_{|\sigma| \leq r} a_{\sigma} D^{\sigma}$ has constant coefficients and where $P(x, D)=\sum_{|\sigma| \leq 1} b_{\sigma}(x) D^{\sigma}$ is a first order operator with $C^{\infty}$-coefficients, which satisfy $\sup _{x}\left|D^{\alpha} b_{\sigma}(x)\right| \leq C_{a}$. Then $L(x, D)$ is essentially maximal.

Proof. One sees that all the assumptions of Theorem 3.5 hold, when we choose $g_{1}(x, \xi) \equiv$ $1, g_{2}(x, \xi)=1+|\xi|$ and $m(x, \xi)=1+|\xi|, \bar{m}(x, \xi)=(1+|\xi|)^{r} \mid$

3.3 For operators of "higher order" we need some additional assumptions on $L(x, \xi)$ to obtain essential maximality. We restrict our considerations (for simplicity) to a special metric. Recall that, for the metric (2.6), one has $h=\frac{1}{9192}$.

Theorem 3.8. Let

$$
g_{(x, \xi)}(y, \eta)=\frac{|y|^{2}}{g_{1}^{2}(x, \xi)}+\frac{|\eta|^{2}}{g_{2}^{2}(x, \xi)}
$$

be a $\sigma$-temperate Riemannian metric, where $g_{1}$ and $g_{2}$ obey (3.2). Assume that there exist $N>0$ and a symbol $P(\cdot, \cdot) \in S\left(\left(g_{1} g_{2}\right)^{N}, g\right)$ such that (with $\gamma>0$ )

$$
|P(x, \xi)| \geq \gamma\left(g_{1} g_{2}\right)^{N}(x, \xi) \text { for all }(x, \xi) \in \mathrm{R}^{2 n} .
$$

Let $L(\cdot, \cdot) \in C^{\infty}\left(\mathbf{R}^{2 n}\right)$ be a symbol such that, with $c, C>0$,

$$
c \leq|L(x, \xi)| \leq C\left(g_{1} g_{2}\right)^{N}(x, \xi)
$$

and

$$
\left|\left(D_{x}^{\alpha} D_{\xi}^{\beta} L\right)(x, \xi)\right| \leq C_{\alpha, \rho}|L(x, \xi)| g_{1}^{-|\alpha|}(x, \xi) g_{2}^{-|\beta|}(x, \xi)
$$

for all $(x, \xi) \in \mathbf{R}^{2 n}$. Then $L(x, D)$ is essentially maximal.

Proof. Part A. Applying the symbolic calculus of Section 2 one sees that there exist $Q(\cdot, \cdot) \in$ $S(1, g)$ and $R_{1}(\cdot, \cdot), R_{2}(\cdot, \cdot) \in S\left(h^{N}, g\right)$ such that

$$
\begin{aligned}
& L \circ Q=I-R_{1} \\
& Q \circ L=I-R_{2} .
\end{aligned}
$$

We sketch the proof of the relations $\left(3.17\right.$ - 3.18). Due to $(3.16)$ we see that $P_{1}(\cdot, \cdot)=1 / L(\cdot, \cdot) \in$ $S(1, g)$. In view of Theorem 2.8 we get

$$
\left(L \circ P_{1}\right)(x, \xi)=1+\sum_{0<|\alpha| \leq N} \frac{1}{\alpha !} \partial_{\xi}^{\alpha} L(x, \xi) D_{x}^{\alpha}\left(\frac{1}{L(x, \xi)}\right)+\tilde{R}_{1}(x, \xi),
$$

where $\tilde{R}_{1}(\cdot, \cdot) \in S(h, g)$ (note that, by $(3.15) \cdot(3.16), L(\cdot, \cdot) \in S\left(h^{-N}, g\right)$ ). By $(3.16)$ the sum

$$
\sum_{0<|a| \leq N} \frac{1}{\alpha !} \partial_{\xi}^{\alpha} L(x, \xi) D_{x}^{\alpha}\left(\frac{1}{L(x, \xi)}\right)
$$

belongs to $S(h, g)$. Hence one sees that there exists $R_{1}(\cdot, \cdot) \in S(h, g)$ such that

$$
\left(L \circ P_{1}\right)(x, \xi)=1-R_{1}(x, \xi) \text {. }
$$

Define $\tilde{P}_{2}(x, \xi)=R_{1}(x, \xi) / L(x, \xi)$. Then we find that

$$
\begin{aligned}
{\left[L \circ\left(P_{1}+\bar{P}_{2}\right)\right](x, \xi) } & =\left(L \circ P_{1}\right)(x, \xi)+\left(L \circ \dot{P}_{2}\right)(x, \xi) \\
& =1-R_{1}(x, \xi)+R_{1}(x, \xi)-R_{2}(x, \xi),
\end{aligned}
$$


where

$$
R_{2}(x, \xi)=-\sum_{0<|\alpha| \leq N} \frac{1}{\alpha !} \partial_{\xi}^{\alpha} L(x, \xi) D_{x}^{\alpha}\left(\frac{R_{1}(x, \xi)}{L(x, \xi)}\right)+\dot{R}_{2}(x, \xi),
$$

in which $\tilde{R}_{2}(\cdot, \cdot) \in S\left(h^{2}, g\right)$. By $(3.16)$ the sum

$$
\sum_{0<|\alpha| \leq N} \frac{1}{\alpha !} \partial_{\xi}^{\alpha} L(x, \xi) D_{x}^{\alpha}\left(\frac{R_{1}(x, \xi)}{L(x, \xi)}\right)
$$

belongs to $S\left(h^{2}, g\right)$ and so

$$
\left(L \circ P_{2}\right)(x, \xi)=1-R_{2}(x, \xi),
$$

where $P_{2}(\cdot, \cdot):=P_{1}(\cdot, \cdot)+\tilde{P}_{2}(\cdot, \cdot) \in S(1, g)$ and $R_{2}(\cdot, \cdot) \in S\left(h^{2}, g\right)$. Generally, by induction one gets that, for any $m \in \mathrm{N}$, there exist $P_{m}(\cdot, \cdot) \in S(1, g)$ and $R_{m}(\cdot, \cdot) \in S\left(h^{m}, g\right)$ such that

$$
\left(L \circ P_{m}\right)(x, \xi)=1-R_{m}(x, \xi) \text {. }
$$

A same kind of construction implies that, for any $m \in \mathrm{N}$, there exist $Q_{m}(\cdot, \cdot) \in S(1, g)$ and $S_{m}(\cdot, \cdot) \in S\left(h^{m}, g\right)$ such that

$$
\left(Q_{m} \circ L\right)(x, \xi)=1-S_{m}(x, \xi) .
$$

Due to (3.19) - (3.20) we have (with $m=2 N$ )

$$
Q_{2 N} \circ L \circ P_{2 N}=Q_{2 N}-Q_{2 N} \circ R_{2 N} \quad \text { and } \quad Q_{2 N} \circ L \circ P_{2 N}=P_{2 N}-S_{2 N} \circ P_{2 N}
$$

and then $P_{2 N}(x, \xi)=Q_{2 N}(x, \xi)+T_{2 N}(x, \xi)$ where $T_{2 N}(\cdot, \cdot) \in S\left(h^{2 N}, g\right)$. Combining the above results we obtain

$$
L \circ P_{2 N}=I-R_{2 N}
$$

and

$$
P_{2 N} \circ L=\left(Q_{2 N}+T_{2 N}\right) \circ L=I-S_{2 N}+T_{2 N} \circ L=I-T_{N},
$$

where $r_{N}(\cdot, \cdot) \in S\left(h^{N}, g\right)$. This proves $(3.17)-(3.18)$.

Similarly as with (3.17) $-(3.18)$ one gets from (3.14) that there exist symbols $q(\cdot, \cdot), r(\cdot, \cdot) \in$ $S\left(h^{N}, g\right)$ such that

$$
q \circ P=I-r .
$$

Part B. In virtue of Theorem $2.7\|(L \circ q) \varphi\|_{0} \leq C\|\varphi\|_{0}$ and so (again by Theorem 2.7)

$$
\begin{aligned}
\|L(x, D) \varphi\|_{0} & =\|L(x, D)((q(x, D) \circ P(x, D)) \varphi+r(x, D) \varphi)\|_{0} \\
& \leq\|P(x, D) \varphi\|_{0}+C^{\prime}\|\varphi\|_{0}
\end{aligned}
$$

for all $\varphi \in S$. Similarly one gets (for $j=1,2$ )

$$
\left\|\left(P(x, D) \circ R_{j}(x, D)\right) \varphi\right\|_{0} \leq C\|\varphi\|_{0}
$$

for all $\varphi \in S$. The inequalities (3.22) - (3.23) imply

$$
R\left(\tilde{R}_{1}\right) \cup R\left(\tilde{R}_{2}\right) \subset D(\tilde{L})
$$

where $R\left(\bar{R}_{j}\right)$ is the range of $\tilde{R}_{j}$. From (3.17) we get

$$
R(\bar{Q}) \subset D(\bar{L})
$$


Part C. Let $u$ be in $D\left(L^{\prime \bullet}\right)$ and let $L^{\prime-} u=f$. Then one gets by (3.18)

$$
\left\langle f, Q^{\prime} \varphi\right\rangle_{0}=\left\langle u, L^{\prime}\left(Q^{\prime} \varphi\right)\right\rangle_{0}=\left\langle u,(Q \circ L)^{\prime} \varphi\right\rangle_{0}=\langle u, \varphi\rangle_{0}-\left\langle u, R_{2}^{\prime} \varphi\right\rangle_{0}
$$

and so $f \in D\left(Q^{\prime *}\right)$ and $Q^{\prime *} f=u-R_{2}^{\prime *} u$. Since $Q$ and $R_{2}$ are bounded in $L_{2}$, one has $Q^{\prime *}=\bar{Q}$ and $R_{2}^{\prime *}=\bar{R}_{2}$. Hence, by (3.24) $\cdot(3.25), u=\bar{Q}_{f}+\bar{R}_{2} u \in D(\tilde{L})$ and $\tilde{L}_{u}=L^{\prime *} u=f$. This completes the proof $I$

Especially, one gets from Theorem 3.8 that the partial differential operator $L(x, D)=$ $\sum_{|\sigma| \leq r} a_{\sigma}(x) D^{\sigma}$ obeying (with $c>0$ )

$$
c \leq|L(x, \xi)| \text { for all }(x, \xi) \in \mathrm{R}^{2 n}, \quad \sup _{x}\left|D_{x}^{o}(x)\right| \leq C_{0} \text { for all }|\sigma| \leq 1
$$

and

$$
\left|\left(D_{x}^{a} D_{\xi}^{\beta} L\right)(x, \xi)\right| \leq C_{\alpha, \beta}|L(x, \xi)|(1+|\xi|)^{-\delta}
$$

with some $\delta \in(0,1]$, is essentially maximal (choose $g_{1}=1, g_{2}(x, \xi)=(1+|\xi|)^{\delta / r}$ and $P(x, \xi)=$ $\left.\left(1+|\xi|^{2}\right)^{r / 2}\right)$.

Remark 3.9. (A) Suppose that $g_{1}$ and $g_{2}$ (which appear in the Riemannian metric $\left\{g_{(x, \xi)}\right\}$ ) obey $g_{1} \in S\left(g_{1}, g\right)$ and $g_{2} \in S\left(g_{2}, g\right)$, that is, $g_{j} \in C^{\infty}\left(\mathbf{R}^{2 n}\right)$ such that

$$
\left|D_{x}^{\alpha} D_{\xi}^{\beta} g_{j}(x, \xi)\right| \leq C_{\alpha, \beta g}(x, \xi) g_{1}^{-|\alpha|}(x, \xi) g_{2}^{-|\beta|}(x, \xi) \quad(j=1,2) .
$$

Then the symbol $P(x, \xi)$ defined by $P(x, \xi)=\left(g_{1} g_{2}\right)^{N}(x, \xi)$ is in $S\left(\left(\dot{g}_{1} g_{2}\right)^{N}, g\right)$ and obeys $(3.14)$. Hence in this case the existence of $P(x, \xi)$ (in Theorem 3.8) is guaranteed. (B) In Theorem 3.8 one may replace the weight function $\left(g_{1} g_{2}\right)^{N}$ by a weight function $m$ for which there are $l \in N$ and $C>0$ such that $m h^{l} \leq C$. We omit this generalization.

\section{Some examples and counter examples}

Combining the Main Theorem of [2] and the Corollary 18.6.11 of [5] we get

Example 4.1. Let $L(x, D)=\sum_{|\sigma| \leq 2} a_{\sigma}(x) D^{\sigma}$ be a second order partial differential operator such that $L^{\prime}(x, D)=L(x, D), L(x, \xi)$ is real-valued, $L(x, \xi) \geq 0$ and that $\left|D_{x}^{\alpha} a_{\sigma}(x)\right| \leq C^{|a|}$ for all $x \in \mathbf{R}^{n}$ and $\alpha \in \mathbf{N}_{0}^{n}$. Then $L(x, D)$ is essentially maximal.

Proof. Due to Corollary 18.6.11 of [5], the operator $L(x, D)$ satisfies the estimate (with $c>0) \quad((L(x, D)+C) \varphi, \varphi\rangle_{0} \geq c\|\varphi\|_{0}^{2}$ for $\varphi \in S$ when $C$ is large enough. Thus the result of [2] implies that $L(x, D)+C$ is essentially maximal and so also $L(x, D)$ is essentially maximal

Example 4.2. Let $L(x, D)=\sum_{|\sigma| \leq k} a_{\sigma}(x) D^{\sigma}$ be a first order linear partial differential operator such that the coefficients $a_{\sigma}$ are first degree polynomials, that is, $a_{\sigma}(x)=\left\langle b_{\sigma}, x\right\rangle+c_{\sigma}$, with $b_{\sigma}=\left(b_{\sigma 1}, \ldots, b_{\sigma n}\right) \in \mathrm{C}^{n}$ and $c_{\sigma} \in \mathrm{C}$. Due to Corollary $3.3 L(x, D)$ is essentially maximal.

Example 4.3. The first order linear partial differential operator (here $n=2$ )

$$
L(x, D)=\left(1+\sin ^{2}\left(\exp x_{1}^{2}\right)\right) D_{2}+1
$$

is essentially maximal. The essential maximality can not be seen by Corollary 3.3 , but may be verified using more direct calculations (we omit the proof). This example shows that the condition (3.8) in Corollary 3.3 need not necessarily be valid for essentially maximal operators. 
Example 4.4. Let $a$ be a (bounded) real-valued function in $C^{\infty}(R)$ such that $a(x) a^{\prime}(x)>0$ for all $x \in R$ (and $a^{\prime}$ is bounded). Define the first order operator by (here $n=1$ )

$$
L(x, D) \varphi=a(x)\left(x^{2}+1\right)^{\alpha} D\left[a(x)\left(x^{2}+1\right)^{\alpha} \varphi\right]+i a(x) a^{\prime}(x)\left(x^{2}+1\right)^{2 a} \varphi \text { for all } \varphi \in S .
$$

Then $L(x, D)$ is not essentially maximal, when $\alpha>\frac{1}{4}$.

Indeed, since the operator $P(x, D) \varphi=a(x)\left(x^{2}+1\right)^{\alpha} D\left[a(x)\left(x^{2}+1\right)^{a} \varphi\right]$ is formally self-adjoint, one sees that $\langle P(x, D) \varphi, \varphi\rangle_{0}$ is real-valued. Hence

$$
\begin{aligned}
\left|\langle L(x, D) \varphi, \varphi\rangle_{0}\right| & =\left.\left|\langle P(x, D) \varphi, \varphi)_{0}+i \int_{\mathbf{R}} a(x) a^{\prime}(x)\left(x^{2}+1\right)^{2 \alpha}\right| \varphi\right|^{2} \mid \\
& \geq \int_{\mathbf{R}} a(x) a^{\prime}(x)\left(x^{2}+1\right)^{2 \alpha}|\varphi|^{2} \\
& \geq \int_{\mathbf{R}} a(x) a^{\prime}(x)|\varphi|^{2} d x
\end{aligned}
$$

and then $N(\tilde{L})=\{0\}$.

On the other hand one sees that the function $u:=\left(x^{2}+1\right)^{-\alpha} \in L_{2} \cap C^{\infty}$ for $\alpha>\frac{1}{4}$ and that

$$
L(x, D) u=-i a(x)\left(x^{2}+1\right)^{\alpha} a^{\prime}(x)+i a(x) a^{\prime}(x)\left(x^{2}+1\right)^{\alpha}=0
$$

and so $N\left(L^{\prime \prime}\right) \neq N(\bar{L})$. Thus $L^{\prime *} \neq \tilde{L}$, as we claimed

We remark that one can choose $a=\arctan : R \rightarrow\left(\frac{1}{2} \pi, \frac{3}{2} \pi\right)$. This example shows that Corollary 3.3 is in some sense strict.

Example 4.5. Let $L(x, D)$ be as in Example 4.4. Since $L(x, D)$ is not essentially maximal, the operator $Q(x, D)=\left(L \circ L^{\prime}\right)(x, D)$ is not essentially maximal (cf. [12]). Hence one sees that there exists a formally self-adjoint, semi-bounded, second order differential operator $Q(x, D)=$ $\sum_{|\sigma| \leq 2} b_{\sigma}(x) D^{\sigma}$ where the growth of the coefficients and their derivatives is at most $(1+|x|)^{\kappa}, \kappa>$ 2 and which is not essentially maximal (cf. Example 4.1).

Example 4.6. Let $\theta$ be areal-valued function in $C_{0}^{\infty}(\mathbf{R})$ such that $\theta(x)=1$, for all $x \in$ $(-1,1)$ and let $a=-\theta x$. Then one sees that $a$ is a real-valued function in $C_{0}^{\infty}(R)$ safisfying $a(0)=0$ and $a^{\prime}(0)=-1$. Define the second order differential operator $L(x, D)$ by (here $n=1$ )

$$
L(x, D) \varphi=\left(a D+(a D)^{\prime}+i\right)[(D+i) \varphi] \text { for all } \varphi \in S .
$$

Since the operators $P=a D+(a D)^{\prime}$ and $Q=D$ are symmetric, one sees that, for all $\varphi \in S$,

$$
\|L(x, D) \varphi\|_{0}=\|(P+i)(D+i) \varphi\|_{0} \geq\|(D+i) \varphi\|_{0} \geq\|\varphi\|_{0} .
$$

Hence we obtain that the kernel $N(\bar{L})$ is $\{0\}$. One the other hand we show that the kernel $N\left(L^{\prime *}\right)$ is not $\{0\}$, which implies that $\tilde{L} \neq L^{\prime *}$.

Let $E$ be in $L_{2}$ defined by $E:=F^{-1}\left(\frac{1}{\xi+i}\right)$ (note that $\int_{R}\left|\frac{1}{\xi+i}\right|^{2} d \xi=\int_{R} \frac{1}{1+\xi^{2}} d \xi<\infty$ and then $E \in L_{2}$; recall that $\mathrm{F}$ is the Fourier transform). Then one sees that (in the distributional sense) $(D+i) E=\delta$. Futhermore we get

$$
\begin{aligned}
& \left\langle E, L^{\prime}(x, D) \varphi\right\rangle_{0}=E\left(\overline{\left.L^{\prime}(x, D) \varphi\right)}=E\left(\overline{((P+i)(\overline{D+i}))^{\prime} \varphi}\right)\right. \\
& =E\left(\overline{\left.(D+i)^{\prime}(P+i)^{\prime} \varphi\right)}=\delta\left(\overline{\left.(P+i)^{\prime} \varphi\right)}\right.\right. \\
& =\left(\overline{\left.(P+i)^{\prime} \varphi\right)}\right)(0)=(\overline{(P-i) \varphi})(0) \text {. }
\end{aligned}
$$


Finally, we find that, for all $\varphi \in S$,

$$
\begin{aligned}
((P-i) \varphi)(0) & =\left[\left(a D+(a D)^{\prime}-i\right) \varphi\right](0)=[a D \varphi+(D a) \varphi+a D \varphi-i \varphi](0) \\
& =2 a(0)(D \varphi)(0)+\left(-i a^{\prime}(0)-i\right) \varphi(0)=0,
\end{aligned}
$$

since $a(0)=0$ and $a^{\prime}(0)=-1$. This completes the proof.

Example 4.7. Similarly, as in Example 4.6 one sees that the pseudo-differential operator (here $n=2$ )

$$
L(x, D) \varphi=\left(a\left(x_{1}\right) D_{1}+\left(a\left(x_{1}\right) D_{1}\right)^{\prime}+i\right)\left[\left(\left(D_{1}^{2}+D_{2}^{2}\right)^{\alpha}+i\right) \varphi\right] \quad(\kappa>1 / 2)
$$

is not essentially maximal in $L_{2}\left(\mathbf{R}^{2}\right)$. Note that the symbol $L(x, \xi)$ of $L(x, D)$ belongs to the Hörmander class $S_{0,1}^{1+2 x}$.

\section{References}

[1] Beals, R. and C. Fepperiman: Spatially inhomogeneous pseudo-differential operators I. Comm. Pure Appl. Math. 27 (1974), 1 - 24.

[2] Devinatz, A.: Essential self-adjointness of certain partial differential operators in $R^{n}$. Proc. Amer. Math. Soc. 60 (1976), 235 - 242.

[3] Goldstein, R.A.: Equality of minimal and maximal extensions of partial differential operators in $L_{p}\left(R^{n}\right)$. Proc. Amer. Math. Soc. 17 (1966), $1031-1033$.

[4] Hess, P.: Über die wesentliche Maximalität gleichmäßig stark elliptischer Operatoren in $L^{2}\left(R^{n}\right)$ Math. Z. 107 (1968), 67 - 70.

[5] Hörmander, L.: The Analysis of Linear Partial Differential Openators III. Berlin: Springer - Verlag 1985.

[6] Kato, T.: Perturbation Theory for Linear Operators. Berlin: Springer - Verlag 1966.

[7] NAOASE, M. and T. UMEDA: On the essential self-adjointness of pseudo-differential operators. Proc. Japan Acad. (Ser. A) 64 (1988), 94 - 97.

[8] REed, M. and B. Simon: Methods of Modern Mathematical Physics I. New York: Academic Press 1972.

[9] REed, M. and B. Simon: Methods of Modern Mathematical Physics II. New York: Academic Press 1975.

[10] Schechter, M.: Spectra of Partial Differential Operators. Amsterdam: North - Holland 1986.

[11] Shubin, M.A.: Pseudo-Differential Operators and Spectral Theory. Berlin: Springer - Verlag 1987.

[12] Steinig, B.: Remarks on the Essential Maximality of Linear Partial Differential Operators. Dissertation. Berlin: Freie Universität 1989

[13] Tervo, J.: On the maximal and minimnal realizations of linear pseudo-differential operators. Comm Phys.-Math. 75 (1986), 16 pp.

[14] Tervo, J.: On the realizations related to Weyl operators. Aequ. Math. 40 (1990), $201-234$. 